\title{
Feasibility of Metatranscriptome Analysis from Infant Gut Microbiota: Adaptation to Solid Foods Results in Increased Activity of Firmicutes at Six Months
}

\author{
Floor Hugenholtz, ${ }^{1}$ Jarmo Ritari, ${ }^{2}$ Lotta Nylund, ${ }^{3}$ Mark Davids, ${ }^{4}$ \\ Reetta Satokari, ${ }^{2,3,5}$ and Willem M. de Vos ${ }^{1,2,5}$ \\ ${ }^{1}$ Laboratory of Microbiology, Wageningen University, Dreijenplein 10, 6703 HB Wageningen, Netherlands \\ ${ }^{2}$ Department of Basic Veterinary Medicine, University of Helsinki, Helsinki, Finland \\ ${ }^{3}$ Functional Foods Forum, University of Turku, Turku, Finland \\ ${ }^{4}$ Laboratory of Systems and Synthetic Biology, Wageningen University, Dreijenplein 10, 6703 HB Wageningen, Netherlands \\ ${ }^{5}$ RPU Immunobiology, Department of Bacteriology and Immunology, University of Helsinki, Helsinki, Finland
}

Correspondence should be addressed to Willem M. de Vos; willem.devos@wur.nl

Received 12 April 2017; Revised 17 June 2017; Accepted 4 July 2017; Published 24 August 2017

Academic Editor: Barbara H. Iglewski

\begin{abstract}
Copyright ( 2017 Floor Hugenholtz et al. This is an open access article distributed under the Creative Commons Attribution License, which permits unrestricted use, distribution, and reproduction in any medium, provided the original work is properly cited.
\end{abstract}

\begin{abstract}
Newborns are rapidly colonized by microbes and their intestinal tracts contain highly dynamic and rapidly developing microbial communities in the first months of life. In this study, we describe the feasibility of isolating mRNA from rapidly processed faecal samples and applying deep RNA-Seq analysis to provide insight into the active contributors of the microbial community in early life. Specific attention is given to the impact of removing rRNA from the mRNA on the phylogenetic and transcriptional profiling and its analysis depth. A breastfed baby was followed in the first six months of life during adaptation to solid food, dairy products, and formula. It was found that, in the weaning period, the total transcriptional activity of Actinobacteria, mainly represented by Bifidobacterium, decreased while that of Firmicutes increased over time. Moreover, Firmicutes and Actinobacteria, including the canonical Bifidobacteria as well as Collinsella, were found to be important contributors to carbohydrate fermentation and vitamin biosynthesis in the infant intestine. Finally, the expression of Lactobacillus rhamnosus-like genes was detected, likely following transfer from the mother who consumed L. rhamnosus GG. The study indicates that metatranscriptome analysis of the infant gut microbiota is feasible on infant stool samples and can be used to provide insight into the core activities of the developing community.
\end{abstract}

\section{Background}

After birth, newborns are rapidly colonized by intestinal microbiota originating from the surrounding environment, including maternal faecal microbes and vaginal or skin species, depending on the mode of delivery $[1,2]$. It is generally assumed that the first colonizers of the infant intestine are facultative anaerobes, such as Streptococcus, Enterococcus and Lactobacillus spp., and Escherichia coli [3, 4], followed by anaerobic bacteria such as Bifidobacterium, Clostridium, and Ruminococcus spp. [5, 6]. Defining a normal intestinal microbiota at early age is challenging, since the composition and temporal patterns of the microbial communities vary widely among infants. During maturation the variation among infants becomes smaller when obtaining a more adult-like microbiota composition, but this has recently been found to take over 5 years of time [7-9]. Bifidobacterium and to a lesser extent Bacteroides are considered the predominant bacterial genera colonizing the early infant gut when the infant is breastfed as these bacteria have the rather unique capacity to degrade human milk oligosaccharides [10]. Introduction of solid foods significantly alters the gut microbiota, switching the microbial composition to be more dominated by Bacteroides and Clostridium spp. [7, 9, 11]. The increase in abundance of the phyla Firmicutes and Bacteroidetes after weaning is an indication of the adaptation to a more complex $\operatorname{diet}[6,7]$. 
TABLE 1: Overview of the infant diet.

\begin{tabular}{lccc}
\hline Sample code & T1 & T2 & \\
\hline Age (days) & 131 & 165 & T3 \\
Breast milk & Exclusively & Yes & No \\
Formula & No & Yes & Yes \\
Potato \& roots & First baby food & Yes & Yes \\
Fruits \& berries & No & Yes & Yes \\
Vegetables & No & Yes & Yes \\
Grain products & No & Yes & Yes \\
Meat/chicken/fish/eggs & No & No & Yes \\
Milk \& dairy products & No & & Yes \\
\hline
\end{tabular}

Various culturing approaches have shown that there is vertical transmission of specific bacteria from mother to baby. This was shown elegantly for endogenous Bifidobacteria [12] as well as probiotic bacteria consumed by the mother that in the case of Lactobacillus rhamnosus GG were found in the baby $[13,14]$. Culture-independent evidence for maternal transmission of early life microbiota is limited, notably as the baby microbiota differs so much from that of the mother. In some cases signatures of parental microbes have been described to be present in the infant microbiome [15] and recently it was reported that the microbiota in children at 3 years of age shows signatures present similar to their own mother and were not seen in between children and unrelated mothers [16].

While considerable attention has been given to the compositional development during early life, only recently high throughput omics-based approaches have been applied. A recent study analysed the faecal metagenome during the first year of life and observed striking differences between vaginal and C-section delivered infants [7]. Moreover, some early studies addressed the transcriptome of Bifidobacteria in the intestinal tract of babies that were breastfed or on a formuladiet and showed differential expression of genes involved in sugar catabolism, exopolysaccharide production, or folate biosynthesis [17]. Moreover metatranscriptome analysis in infants and their mothers showed differences in expression of higher capacity of mucin utilization, higher capacity folate biosynthesis, and decreased starch degradation in infants [18]. Global metatranscriptome analysis in the GI tract microbiota could enable the elucidation of the specific functional roles microbes have in this complex community. Initial metatranscriptome studies in the human large intestine revealed that different functions are expressed between individuals while core functions of the microbiota appeared to be consistently expressed among individuals [19-21]. These findings imply that metatranscriptomics could provide insight into the differential activity profiles in the gut microbiota, enabling the reconstruction of the metabolic activity profile of microbial communities.

In this study we describe the feasibility of using deep RNA-Seq analysis to get further insight in the active contributors of the microbial community in early life. Specific attention is given to the rapid sampling and impact of removing rRNA from the mRNA on the phylogenetic and transcriptional profiling and its analysis depth. A breastfed baby was followed in the first six months of life during adaptation to solid food, dairy products, and formula. The results indicate that Bifidobacterium is an active member of the community and over time various members of the Firmicutes become more active that are involved in vitamin production and sugar metabolism at 6 months.

\section{Methods}

2.1. Subject, Dietary Information, and Sampling. One vaginally born, breastfed, Finnish baby girl was followed during the introduction of first solid foods into the diet. Faecal samples were collected at three time points, specifically at the ages of 131, 165, and 171 days. These samples were given with consent of the mother and ethics are within national and international regulation. The time points and number of samples were taken for practical reasons as the faecal samples were taken at home and immediately processed in RNAlater as to preserve the mRNA as good as possible. The infant did not receive any probiotic supplementation, but her mother consumed dairy products containing Lactobacillus rhamnosus GG. Both infant and her mother were healthy and did not receive any antibiotics during the study period. At the first time point (131 days), the infant consumed exclusively breast-milk and some mashed potato and roots as the first baby food, which was extended in the second time point (165 days) to fruits, vegetables, and grain and meat products. Before the last time point the infant was also introduced to dairy products and formula milk (Table 1).

\subsection{RNA Extraction, rRNA Removal, cDNA Synthesis, Library} Preparation, and Illumina MiSeq 2500 Sequencing. Fresh faecal samples were collected and immediately processed as described previously [22]. For this purpose, one ml RNAlater was added to each gram of sample and stored at $-70^{\circ} \mathrm{C}$ until later processing. The amount of faecal material used for the RNA extraction was $6.81 \mathrm{~g}$ for $\mathrm{T} 1,4.12 \mathrm{~g}$ for $\mathrm{T} 2$, and $4.42 \mathrm{~g}$ for $\mathrm{T} 3$, respectively. Total RNA was extracted as described before using the Macaloid procedure [23] and final RNA concentrations were measured with the NanoDrop 1000. Here we obtained $35.09,38.04$, and $31.82 \mu \mathrm{g}$ of total RNA for T1, T2, and T3, respectively. From sample T2, $5 \mu \mathrm{g}$ of RNA was also used for rRNA removal by using the 
Ribo-Zero kit (Epicentre), which is a magnetic beads-based rRNA hybridization technique to remove the 23S, 16S, and $5 S$ rRNA from the sample. One sample was taken only, to see the effect of the rRNA removal in the sequencing results. This portion was further named T2_M, while the sample containing total RNA was labelled T2_T. $500 \mathrm{ng}$ of total RNA from the samples T1, T2_T, and T3 and due to the rRNA removal $244 \mathrm{ng}$ of $\mathrm{mRNA}$ of T2_M was used for cDNA synthesis and library construction (TrueSeq RNA Sample Preparation Kit, Illumina, San Diego, CA). Due to the quality differences, slightly different lengths were taken for the sequencing: T1: 300-700 bp, T2_T: 300-570 bp, T2_M: 300-500 bp, and T3: 300-500 bp. Sequencing was performed using the Illumina MiSeq instrument on $2 \times 150$ bp paired end mode. Between 3 and 4 million reads were obtained per sample.

2.3. RNA-Seq Data Processing. The raw reads data are available in the MG-RAST server under the following accession codes: 4621794.3, 4621795.3, 4621796.3, 4621797.3, 4621798.3, $4621799.3,4621800.3$, and 4621801.3 . After fastq quality filtering reads were taxonomically assigned by matching against a human intestinal 16S rRNA database (https:/github.com/ microbiome/HITdb, Ritari et al. unpublished) at 97\% identity threshold. For the mRNA analysis rRNA reads were rapidly filtered from the samples using SortmeRNA (version 1.2), after which the remaining reads were assembled using idba_ud using the pipeline described previously [24]. Essential single copy genes (ESCG), identified using HMM search (http://hmmer.org/), were taxonomically classified using MEGAN after being aligned against full NR database. The remainder of the proteins was taxonomically classified by aligning them against all proteins in the NR database belonging to members of nine identified bacterial orders. Functional annotation was performed to all predicted protein sequences by assignment of KEGG orthology identifiers using the KEGG KAAS server. Expression levels of the predicted ORFs were determined by aligning the reads against the assembly using bowtie 2 and counting reads mapped to each ORF using BEDtools.

\section{Results and Discussion}

3.1. In Vitro rRNA Removal Increases Functional Transcription Depth. Since rRNA is known to dominate the composition of total bacterial RNA, we employed an in vitro rRNA removal procedure to evaluate its effect on the content and accuracy of the sequenced RNA pool. Thus from one of the samples (T2_M), rRNA was removed prior to the RNASeq analysis to enrich for mRNA, while in all other samples total RNA was sequenced. Thus, it allowed the comparison of the same sample with and without rRNA removal (i.e., T2_T and T2_M). After quality filtering, a total number of approximately 2.5 million reads were obtained in all samples (Figure 1(a)). However, when analysing these, it was observed that reads derived from rRNA were dominating in samples where in vitro rRNA removal was not applied; in silico filtering of rRNA sequences by means of matching to $5 \mathrm{~S}, 16 \mathrm{~S}$, and $23 \mathrm{~S}$ rRNA gene databases removed about $99 \%$ of reads,

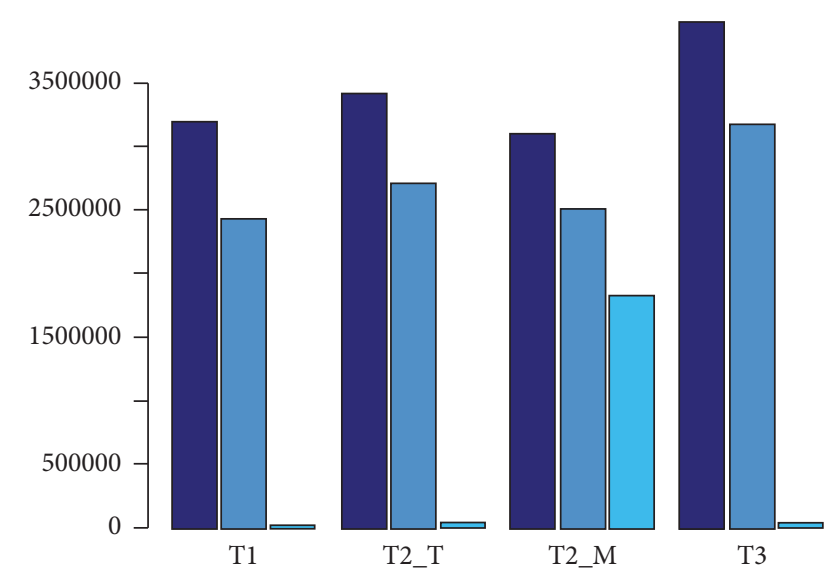

(a)

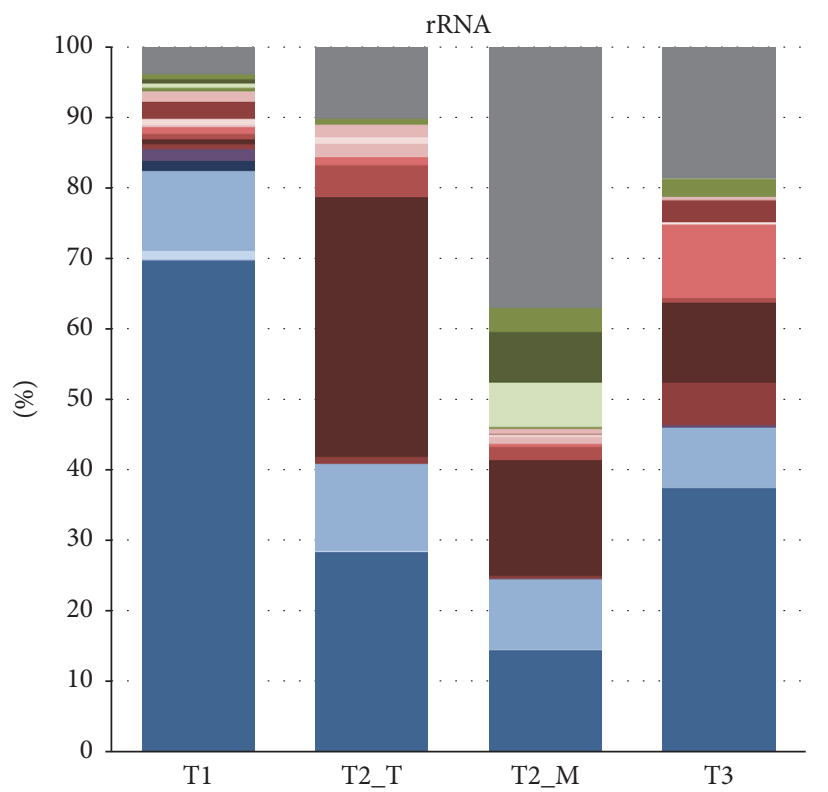

- Dorea

- Lachnoclostridium

- Blautia

- Clostridium

- Lactobacillus

- Propionibacterium

- Collinsella

- Actinomyces

- Bifidobacterium

(b)
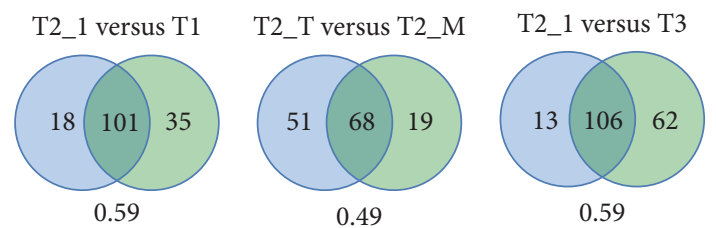

(c)

FIGURE 1: (a) Numbers of reads at different stages of in silico filtering. Raw reads (dark blue), after quality filtering (medium blue) and after quality and rRNA filtering (light blue). (b) Taxonomic profiles based on 16S rRNA. (c) Numbers of shared genera between T2_T and other samples. The Jaccard index below the Venn diagrams shows the relationship between shared and all genera. 
with the exception of sample T2_M where only about $28 \%$ of reads were removed. This showed that T2_M contained much less rRNA than untreated samples and confirmed the efficiency of the in vitro rRNA removal procedure. As a consequence, for sample T2_M, the number of filtered mRNA reads was approximately 250 -fold higher than that of the untreated samples.

\subsection{Impact of rRNA Removal on $16 S$ rRNA Based Taxonomic} Profiles. Comparison of taxonomical profiles of samples with and without in vitro rRNA removal (T2_T versus T2_M) showed a significant difference between the samples (Figure 1(b)). Indeed, the difference between T2_T and T2_M was more pronounced than the difference between samples $\mathrm{T} 2{ }_{-} \mathrm{T}$ and $\mathrm{T} 3$, representing different time points. The difference was especially evident in the abundance of Bifidobacterium spp. that showed an approximately twofold reduction in sample T2_M as compared to the identical but not treated sample T2_T (Figure 1(b)). Furthermore, the amount of $16 \mathrm{~S}$ rRNA sequences of Blautia was also approximately 2 -fold reduced, while that of Proteobacteria increased over 15-fold in T2_M, altogether indicating a strong effect of the rRNA removal by the kit. In addition, the number of common genera between the samples $\mathrm{T} 2{ }_{-} \mathrm{T}$ and $\mathrm{T} 2 \mathrm{M}$ was lower than that between other samples; T2_T had more in common with T1 and T3 than with T2_M (Figure 1(c)). Moreover, comparing the log relative abundances between $\mathrm{T} 2 \_\mathrm{T}$ and $\mathrm{T} 2 \_\mathrm{M}$ indicated that there were several noncorrelating genera, which were abundant in T2_M but rare in T2_T (Supplementary Figure 1 in Supplementary Material available online at https://doi.org/10.1155/2017/9547063).

Altogether, the results indicate that the used in vitro rRNA removal procedure causes bias to the taxonomic composition, most likely because the procedure does not target all taxonomic groups equally. Thus it generates taxonomic profiles with different bacterial content and abundances when compared to untreated samples. This has also been observed for other rRNA removal methods in a study with a synthetic community consisting of 5 genera that are not common inhabitants of the intestinal tract. [25]. Here, we confirm and extend this analysis and show that the rRNA removal using the Ribo-Zero kit (Epicentre) specifically reduces the rRNA fraction of the Firmicutes and Actinobacteria from the intestinal microbiome of the infant, while increasing that of the Proteobacteria. Hence, taxonomic analysis based on 16S rRNA sequences from in vitro treated material should be taken with caution, even though there would be enough reads left after the removal.

3.3. Metatranscriptome. For further analysis at mRNA-level, the data was filtered to remove rRNA sequences, adapter sequences, and poor quality reads. SortMeRNA [26] was used to rapidly filter out rRNA sequences using the precompiled databases for eukaryotes, bacteria, and archaea. To determine the function and taxonomy of the mRNA reads, they were merged and de novo assembled into larger contigs, creating a single contig reference set for all samples. A total of 11558 contigs larger than $300 \mathrm{bp}$ could be assembled with an overall length of 9,843,629 bases. These contigs encoded a total of
TABLE 2: Sequencing, assembly, and mapping information of the samples.

\begin{tabular}{lcccc}
\hline & $\begin{array}{c}\text { Total reads } \\
\text { (pairs) }\end{array}$ & $\begin{array}{c}\text { mRNA reads } \\
\text { (pairs) }\end{array}$ & $\begin{array}{c}\text { Mapped } \\
\text { (single) }\end{array}$ & \% mapped \\
\hline T1 & 1591215 & 6229 & 3801 & 30.5 \\
T2_T & 1710009 & 13749 & 17570 & 63.9 \\
T2_M & 1550435 & 1084270 & 1480261 & 68.3 \\
T3 & 1986769 & 15661 & 15657 & 50.0 \\
\hline
\end{tabular}

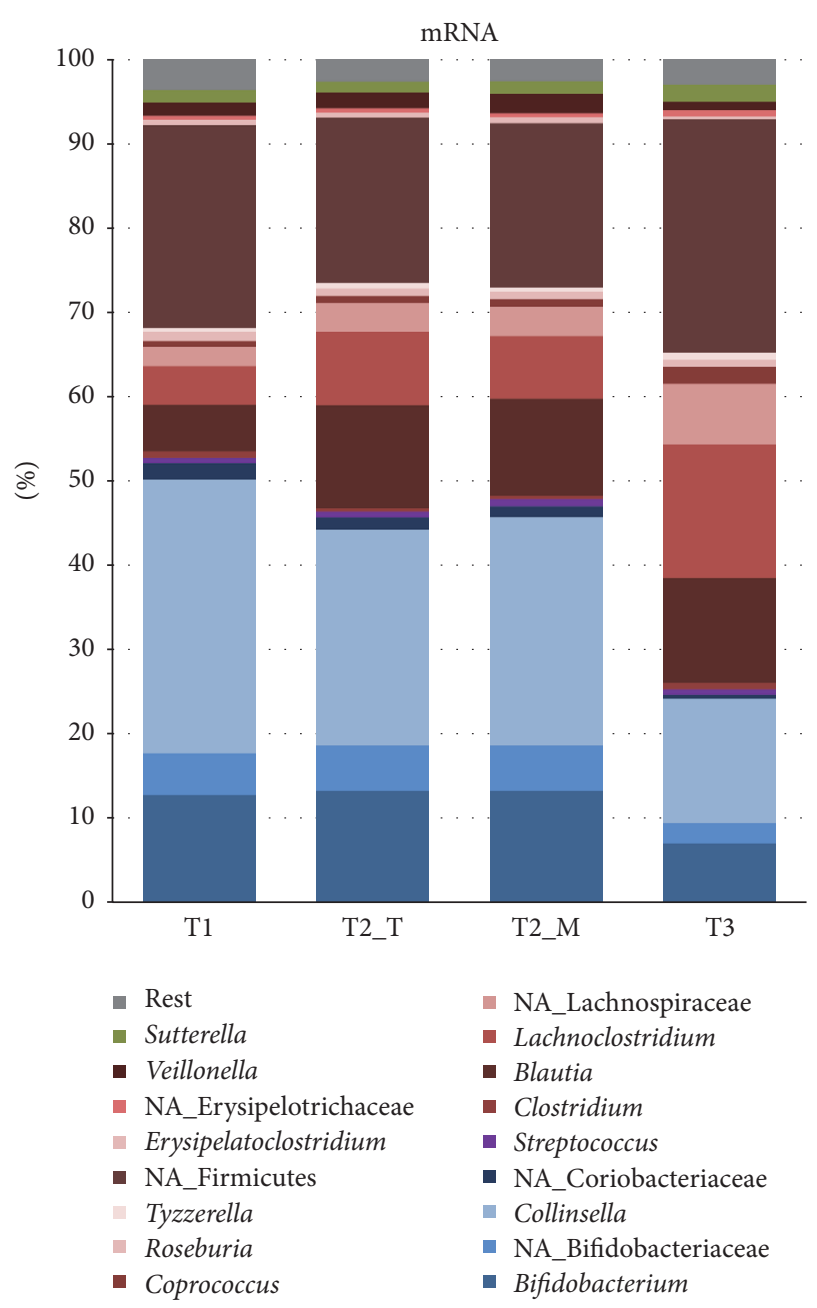

Figure 2: Taxonomic profiles based on mRNA.

16,422 predicted open reading frames. Altogether 30 to $68 \%$ of the mRNA reads could be mapped and assigned to proteinencoding gene regions (Table 2).

The protein assigned mRNA reads were used for a detailed taxonomic analysis (Figure 2). As the mapping is not without bias and quite variable (Table 2), we observed various differences in the phylogenetic composition of the baby samples based the 16S rRNA (Figure 2) and mRNA data (Figure 2). These differences in abundances may also be explained by varying gene expression levels that do not necessarily reflect the real abundances of underlying bacterial groups. Remarkably, the phylogenetic composition, based on 


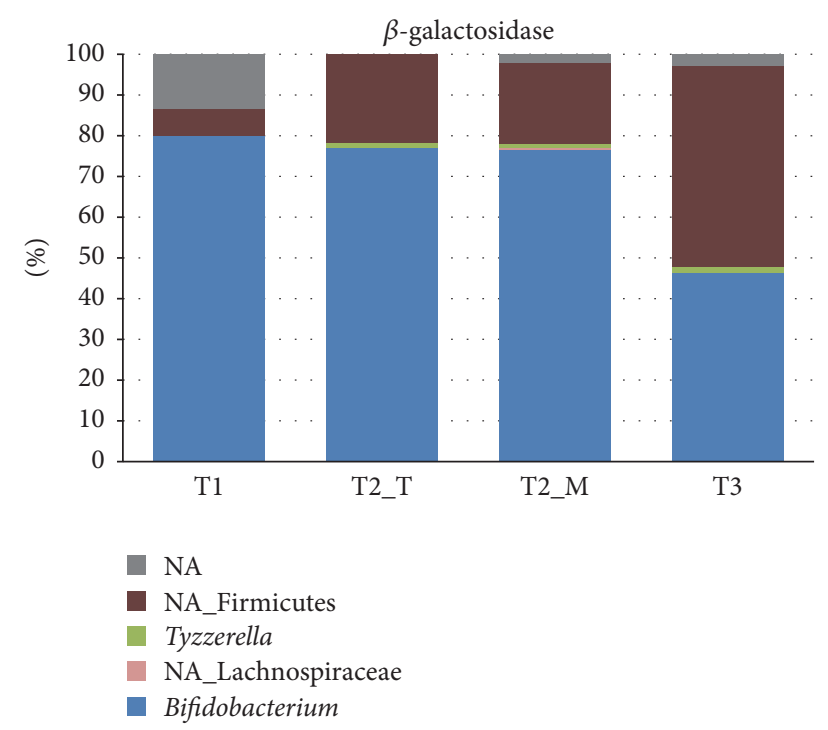

Figure 3: Taxonomic profiles based on expression of the $\beta$ galactosidase gene.

the mapped mRNA, appeared to be not affected by the in vitro rRNA removal as indicated by a Pearson correlation coefficient of T2_T and T2_M samples of 0.99 (Supplementary Figure 2). This suggests that the rRNA removal is likely to preserve mRNA levels with high fidelity.

The main taxonomic groups expressing protein-encoding transcripts belonged to Bifidobacterium, Collinsella, Blautia, Lachnoclostridium, and unidentified Firmicutes (Figure 2). The expression of mRNA derived from Bifidobacterium and Collinsella decreased from T1 to T3, most likely due to the introduction of new solid foods into the infant diet after T1. As the weaning process proceeds, a wider variety of carbon sources becomes available in the infant intestine, thus supporting the establishment of more diverse microbial community. This was demonstrated by the high expression of bifidobacterial $\beta$-galactosidase while the infant received exclusively breast-milk (T1), whereas unidentified Firmicutes were the main producer of this enzyme at T3 when the infant diet included also a variety of solid foods and dairy products (Figure 3). Transcripts derived from Bacteroidetes were hardly found in the dataset. While Bacteroidetes have been reported to be present during weaning $[6,7]$, their absence in 3-month healthy and breastfed babies is not unusual $[18,27]$. As the rRNA-derived composition also indicated a low level of Bacteroidetes (Figure 1(b)), this indicated that the infant's gut microbiota during weaning is still adapting towards more adult-like composition. However we must point out here that we have not obtained specific information on the sampling time during the day and the exact amount and frequency of the food intake; this could change activity patterns considerably and should be taken into consideration in future experiments.

3.4. In-Depth Functional and Taxonomic Distributions of Metabolic Pathways. Metagenome analyses have indicated that the production of vitamins may be one of the benefits of the early life intestinal microbiota [7, 9]. However, evidence based on expression studies is lacking apart from indications of folate biosynthesis by Bifidobacteria in 8-monthold infants [17]. The sequencing depth in sample T2_M was sufficient for determining the taxonomic distribution of the vitamin pathways (Supplementary Figure 3) as well as that of sugar transport and the taxonomic distribution of the glycosylases expression. Remarkably, the expressed genes involved in biosynthesis of the vitamin folate (B9) reflect similar taxonomic distribution as the $\beta$-galactosidase and the overall mRNA expression profiles to some extent, with Bifidobacterium, Collinsella, and Blautia as the main contributors to folate metabolism (Figure 4). Other vitamins such as B6, $\mathrm{B} 7$, and B12 were each produced by quite different organisms, including Blautia, Lachnoclostridium, and Lachnospiraceae, which mainly belonged to phylum Firmicutes. This indicates that other bacteria than the canonical Bifidobacterium spp. contribute to important functions such as vitamin production at 6 months of age.

We were also able to detect in sample T2_M the expression of a large variety of genes for sugar transporters belonging to PEP-dependent phosphotransferase (PTS) or ATP binding cassette $(\mathrm{ABC})$ systems that are characterized by the involvement of multiple proteins (Table 3). Not all transcripts of the transporter module proteins functions were equally expressed but this may be due to differential gene expression or transcript stability. Metagenomic analysis had also revealed the presence of these transporters in infants of 4 and/or 12 months [7]. Altogether, the expression of these transporter genes indicates that a large range of sugars is transported by the gut microbiota in an infant of approximately 6 months old. Moreover, the expression of glycosidase genes specific for the hydrolysis of the transported saccharides was also detected, indicating the functional use of these sugars, including alpha- and beta-glycosidases (altogether denoted as glycosylase genes) (Figure 5; Supplementary Table 1 ). These glycosidase genes had been previously found to be present before the introduction of solid food [6]. While Actinobacteria expressed the largest amount of sugar transport and degradation genes, around $25 \%$ of the transcripts derived from Firmicutes, indicating that members of both phyla are important contributors to the carbohydrate degradation.

3.5. Expression Levels of Genes from Lactobacillus rhamnosus GG. While breastfeeding, the mother consumed dairy products containing Lactobacillus rhamnosus GG (LGG), which is very common in many dairy products and drinks in Finland and has the capacity to strongly interact with human mucus via its protruding pili [28]. Previously, it has been shown that mother-infant transfer of LGG may occur frequently [14]. To study whether we could deduce the presence of LGG or related bacteria in the transcriptome of the studied infant, mRNA reads were mapped to LGG genome at 95\% similarity threshold. This analysis confirmed the expression of LGGlike genes, including these present in variable regions present in LGG but absent in many other L. rhamnosus strains [29]. Comparative analysis revealed significantly more LGG-like genes (\% of total mRNA reads) in sample T1 when compared to samples T2 and T3 (Figure 6). The same result was seen 


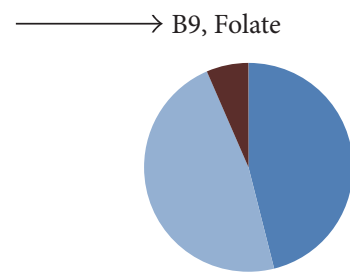

- Bifidobacterium

- Collinsella

- Blautia
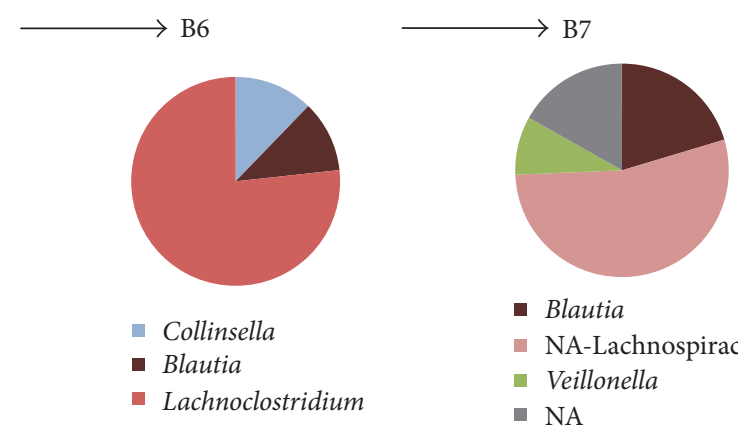

- Blautia

- NA-Lachnospiraceae

- Veillonella

- NA

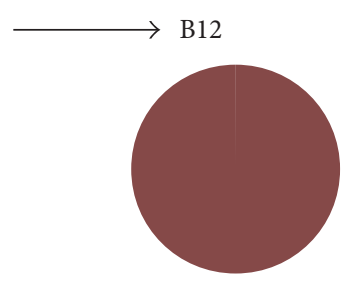

- NA-Clostridiales

\begin{tabular}{llllr}
\hline & B9 & B6 & B7 & B12 \\
Expression level & 55 & 67 & 15 & 29 \\
\hline
\end{tabular}

FIGURE 4: Relative taxonomic profiles based on the final enzyme for the corresponding vitamin metabolism of sample T2_M. KEGG numbers for the final step in the corresponding vitamin pathways for this figure are K00287 (B9, Folate), K00868 (B6); K01012 (B7, Biotin); K02233 (B12).

TABLE 3: Overview of the expressed PTS and ABC transport modules involved in sugar transport in sample T2_M. $p$ values and adjusted $p$ values indicate the likelihood of a KEGG module to be enriched on a background of amongst all detected KEGG orthologs.

(a) PTS transporter modules

\begin{tabular}{lccccc}
\hline Module number & $p$ value & Adjusted $p$ value & $\begin{array}{c}\text { Number of } \\
\text { proteins in } \\
\text { modules }\end{array}$ & $\begin{array}{c}\text { Number of } \\
\text { tran- } \\
\text { scribed } \\
\text { proteins- } \\
\text { encoding } \\
\text { genes }\end{array}$ & Description \\
\hline M00266 & 0 & 0.006 & 3 & 2 & Maltose and glucose-specific II component \\
M00269 & 0 & 0.006 & 3 & 2 & Sucrose-specific II component \\
M00268 & 0 & 0.006 & 3 & 2 & Arbutin-like II component \\
M00273 & 0 & 0.006 & 3 & 2 & Fructose-specific II component \\
M00270 & 0.001 & 0.011 & 4 & 2 & Trehalose-specific II component \\
M00277 & 0.001 & 0.011 & 4 & 2 & N-Acetylgalactosamine-specific II component \\
M00267 & 0.031 & 0.555 & 3 & 1 & N-Acetylglucosamine-specific II component \\
M00282 & 0.031 & 0.555 & 3 & 1 & D-Glucosamine-specific II component \\
M00272 & 0.031 & 0.555 & 3 & 1 & Arbutin-, cellobiose-, and salicin-specific II component \\
M00303 & 0.031 & 0.555 & 3 & 1 & N-Acetylmuramic acid-specific II component \\
M00279 & 0.031 & 0.555 & 3 & 1 & Galactitol-specific II component \\
M00283 & 0.031 & 0.555 & 3 & 1 & Ascorbate-specific II component \\
\hline
\end{tabular}

(b) ABC sugar transporter modules

\begin{tabular}{lccccc}
\hline Module number & $p$ value & Adjusted $p$ value & $\begin{array}{c}\text { Number of } \\
\text { proteins in } \\
\text { modules }\end{array}$ & $\begin{array}{c}\text { Number of } \\
\text { tran- } \\
\text { scribed } \\
\text { protein- } \\
\text { encoding } \\
\text { genes }\end{array}$ & Description \\
\hline M00601 & 0 & 0.009 & 3 & 2 & Putative chitobiose transport system \\
M00219 & 0.001 & 0.018 & 4 & 2 & AI-2 transport system \\
M00211 & 0.025 & 0.48 & 2 & 1 & Putative ABC transport system \\
M00217 & 0.038 & 0.716 & 3 & 1 & D-Allose transport system \\
M00210 & 0.038 & 0.716 & 3 & 1 & Putative ABC transport system \\
M00605 & 0.05 & 0.949 & 4 & 1 & Glucose/mannose transport system \\
M00206 & 0.05 & 0.949 & 4 & Cellobiose transport system \\
M00606 & 0.05 & 0.949 & 4 & 1 & N,N ${ }^{\prime}$-Diacetylchitobiose transport system \\
M00197 & 0.05 & 0.949 & 4 & 1 & Putative fructooligosaccharide transport system \\
\hline
\end{tabular}




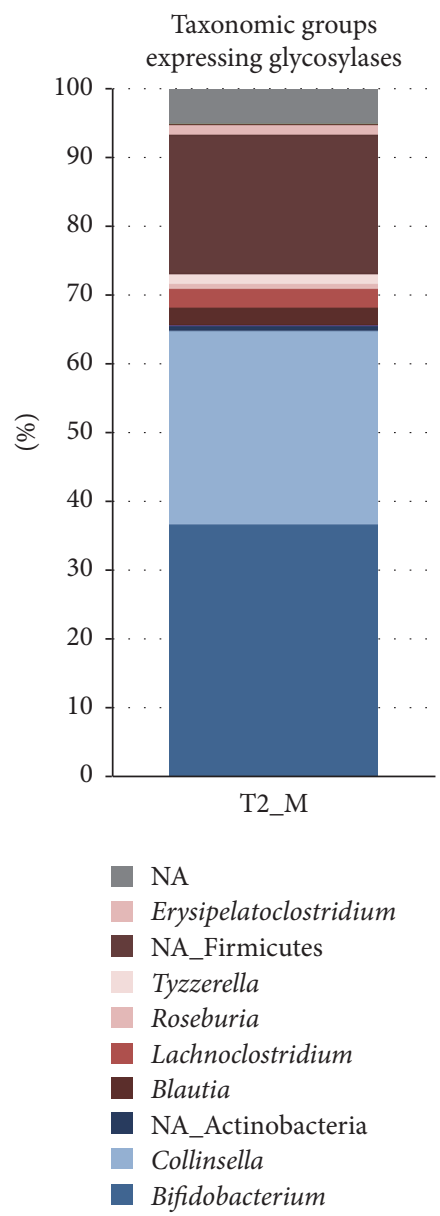

Figure 5: Taxonomic profiles based on expression of the glycosylases genes in sample T2_M.

when mapping rRNA reads from faecal samples to LGG $16 \mathrm{~S}$ rRNA sequence at $97 \%$ similarity. While only a few different LGG-like functions could be detected in the transcriptome of sample T1, in that of sample T2_M, the expression of the LGGlike genes related to glycolysis is clearly visible, indicative of its high activity (Supplementary Figure 4). Since the sample T1 represents the microbiota of an infant with breast-milk as the main source of nutrients and whose mother consumed LGG containing products, it may be speculated that LGG was acquired during or soon after the birth from the mother. At later time points the abundance of LGG-like transcripts dropped, suggesting that LGG would then not permanently colonize the infant intestinal tract ecosystem. However, as this coincides with the introduction of formula and solid food, one may speculate that these factors also contribute to the clearance of LGG.

\section{Conclusions}

We demonstrate here the feasibility of metatranscriptome analysis using high throughput RNA-Seq on the infant gut to get in-depth insight in the active community structure in the weaning period. A relevant technical observation is

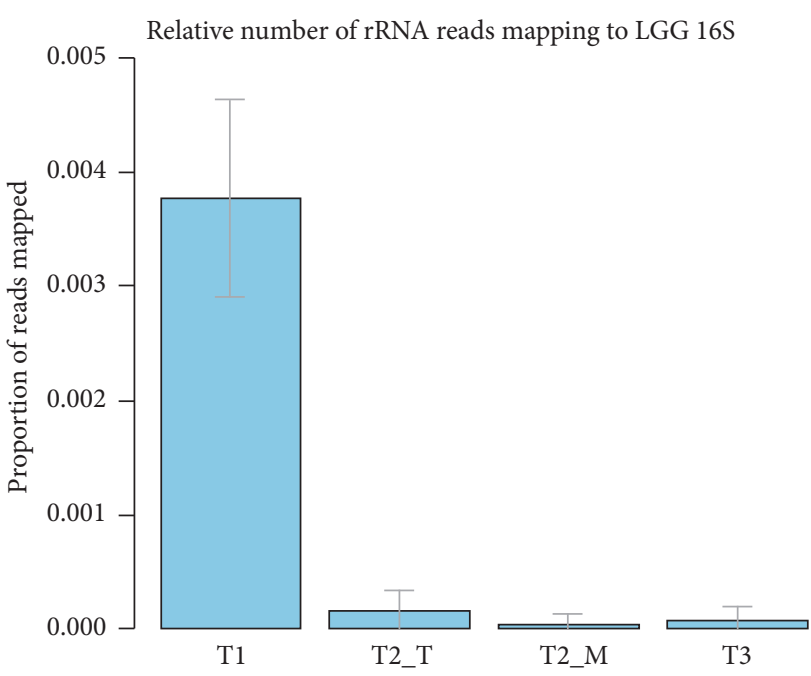

(a)

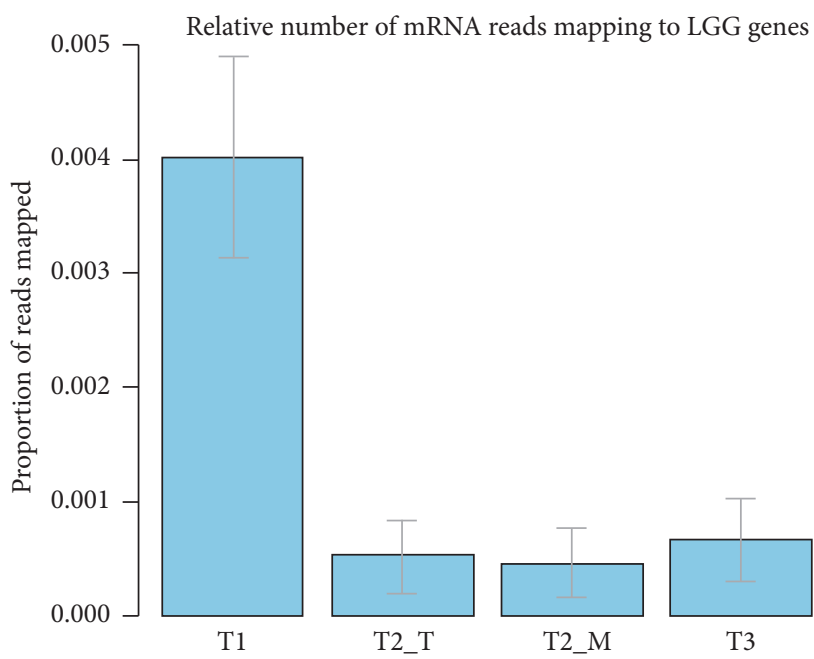

(b)

FIGURE 6: Relative numbers of mRNA (b) and rRNA (a) read mapping to LGG. Error bars represent standard deviations based on binomial sampling.

that rRNA removal with Ribo-Zero kit (Epicentre) has a pronounced effect on the rRNA distribution but not on that of mRNA. It was found that in the weaning period of the breastfed infant a number of major activity changes occurred with a decrease in activity of the Actinobacteria, mainly represented by Bifidobacterium, and an increase in activity of members of the Firmicutes increased in activity over time. Moreover, the Firmicutes and Actinobacteria, including the canonical Bifidobacteria and Collinsella, were found to be important contributors to vitamin production in the infant intestine, including that of vitamin B6, B7, B9, and B12. Finally, the mother consumed the probiotic LGG and we detected LGG-like transcripts in the infant transcriptome, indicating that LGG has been transferred, is an active member at the earliest time point, and is decreasing in abundance and activity during weaning, showing that LGG is not likely to remain a permanent member of the intestinal microbiota. 
The here described approach and pipeline allow for further longitudinal analysis of infant activity to complement infant cohort metagenome studies that are presently developing.

\section{Disclosure}

The abstract of this paper was presented before on the Darmendag (Gutflora day) 2015 in Rotterdam, Netherlands.

\section{Conflicts of Interest}

The authors declare that they have no conflicts of interest.

\section{Authors' Contributions}

Lotta Nylund, Reetta Satokari, and Willem M. de Vos designed the study and Lotta Nylund carried out the RNA extractions and preparation of the sequencing. Mark Davids and Jarmo Ritari preprocessed the RNA-Seq reads and Jarmo Ritari analysed the rRNA-Seq data. Floor Hugenholtz analysed the mRNA-Seq data. Floor Hugenholtz and Willem M. de Vos drafted the manuscript. All authors read and approved the final manuscript.

\section{Acknowledgments}

This research was partly supported by ERC Advanced Grant 250172, MicrobesInside from the European Research Council, the Netherlands Organization for Scientific Research (Spinoza Award and SIAM Gravity Grant 024.002.002), and the Finland Academy of Sciences (141130).

\section{References}

[1] D. M. Chu, J. Ma, A. L. Prince, K. M. Antony, M. D. Seferovic, and K. M. Aagaard, "Maturation of the infant microbiome community structure and function across multiple body sites and in relation to mode of delivery," Nat Med, vol. 23, no. 3, pp. 314-326, 2017.

[2] M. G. Dominguez-Bello, K. M. De Jesus-Laboy, N. Shen et al., "Partial restoration of the microbiota of cesarean-born infants via vaginal microbial transfer," Nature Medicine, vol. 22, pp. 250-253, 2016.

[3] L. Moles, M. Gómez, H. Heilig et al., "Bacterial diversity in meconium of preterm neonates and evolution of their fecal microbiota during the first month of life," PLOS ONE, vol. 8, no. 6, Article ID e66986, 2013.

[4] H. Wopereis, R. Oozeer, K. Knipping, C. Belzer, and J. Knol, "The first thousand days - intestinal microbiology of early life: Establishing a symbiosis," Pediatric Allergy and Immunology, vol. 25, no. 5, pp. 428-438, 2014.

[5] C. F. Favier, E. E. Vaughan, W. M. De Vos, and A. D. Akkermans, "Molecular monitoring of succession of bacterial communities in human neonates," Appl Environ Microbiol, vol. 68, no. 1, pp. 219-226, 2002.

[6] J. E. Koenig, A. Spor, N. Scalfone et al., "Succession of microbial consortia in the developing infant gut microbiome," Proceedings of the National Academy of Sciences of the United States of America, vol. 108, supplement 1, pp. 4578-4585, 2011.
[7] F. Backhed, J. Roswall, Y. Peng, Q. Feng, H. Jia, P. KovatchevaDatchary et al., "Dynamics and stabilization of the human gut microbiome during the first year of life," Cell Host and Microbe, vol. 17, no. 5, pp. 690-703, 2015.

[8] J. Cheng, T. Ringel-Kulka, I. Heikamp-de Jong, Y. Ringel, I. Carroll, and W. M. de Vos, "Discordant temporal development of bacterial phyla and the emergence of core in the fecal microbiota of young children," ISME J, 2015.

[9] T. Yatsunenko, F. E. Rey, M. J. Manary et al., "Human gut microbiome viewed across age and geography," Nature, vol. 486, no. 7402, pp. 222-227, 2012.

[10] A. Marcobal, M. Barboza, E. D. Sonnenburg et al., "Bacteroides in the infant gut consume milk oligosaccharides via mucusutilization pathways," Cell Host and Microbe, vol. 10, no. 5, pp. 507-514, 2011.

[11] N. Ottman, H. Smidt, W. M. de Vos, and C. Belzer, "The function of our microbiota: who is out there and what do they do?" Frontiers in Cellular and Infection Microbiology, vol. 2, p. 104, 2012.

[12] H. Makino, R. Martin, E. Ishikawa et al., "Multilocus sequence typing of bifidobacterial strains from infant's faeces and human milk: Are bifidobacteria being sustainably shared during breastfeeding?" Beneficial Microbes, vol. 6, no. 4, pp. 563-572, 2015.

[13] R. K. Buddington, C. H. Williams, B. M. Kostek, K. K. Buddington, and M. J. Kullen, "Maternal-to-infant transmission of probiotics: concept validation in mice, rats, and pigs," Neonatology, vol. 97, no. 3, Article ID 000253756, pp. 250-256, 2010.

[14] M. Gueimonde, S. Sakata, M. Kalliomaki, E. Isolauri, Y. Benno, and S. Salminen, "Effect of maternal consumption of lactobacillus GG on transfer and establishment of fecal bifidobacterial microbiota in neonates," J Pediatr Gastroenterol Nutr, vol. 42, no. 2, pp. 166-170, 2006.

[15] M. G. Dominguez-Bello, E. K. Costello, M. Contreras et al., "Delivery mode shapes the acquisition and structure of the initial microbiota across multiple body habitats in newborns," Proceedings of the National Academy of Sciences, vol. 107, no. 26, pp. 11971-11975, 2010.

[16] L. Nylund, "Early Life Intestinal Microbiota in Health and in Atopic Eczema," Tech. Rep., 2015.

[17] E. S. Klaassens, R. J. Boesten, M. Haarman et al., "Mixed-species genomic microarray analysis of fecal samples reveals differential transcriptional responses of bifidobacteria in breast- and formula-fed infants," Appl Environ Microbiol, vol. 75, no. 9, pp. 2668-2676, 2009.

[18] F. Asnicar, S. Manara, M. Zolfo, D. T. Truong, M. Scholz, and F. Armanini, "Studying vertical microbiome transmission from mothers to infants by strain-level metagenomic profiling," mSystems, vol. 2, no. 1, pp. e00164-00116, 2017.

[19] C. C. G. M. Booijink, J. Boekhorst, E. G. Zoetendal, H. Smidt, M. Kleerebezem, and W. M. de Vos, "Metatranscriptome analysis of the human fecal microbiota reveals subject-specific expression profiles, with genes encoding proteins involved in carbohydrate metabolism being dominantly expressed," Applied and Environmental Microbiology, vol. 76, no. 16, pp. 5533-5540, 2010.

[20] E. A. Franzosa, X. C. Morgan, N. Segata et al., "Relating the metatranscriptome and metagenome of the human gut," Proceedings of the National Academy of Sciences of the United States of America, vol. 111, no. 22, pp. E2329-E2338, 2014.

[21] M. J. Gosalbes, A. Durbán, M. Pignatelli et al., "Metatranscriptomic approach to analyze the functional human gut microbiota," PLoS ONE, vol. 6, no. 3, Article ID e17447, 2011. 
[22] E. G. Zoetendal, H. G. Heilig, E. S. Klaassens et al., "Isolation of DNA from bacterial samples of the human gastrointestinal tract," Nature protocols, vol. 1, no. 2, pp. 870-873, 2006.

[23] E. G. Zoetendal, C. C. Booijink, E. S. Klaassens et al., "Isolation of RNA from bacterial samples of the human gastrointestinal tract," Nature protocols, vol. 1, no. 2, pp. 954-959, 2006.

[24] M. Davids, F. Hugenholtz, V. Martins dos Santos, H. Smidt, M. Kleerebezem, and P. J. Schaap, "Functional profiling of unfamiliar microbial communities using a validated de novo assembly metatranscriptome pipeline," PLoS One, vol. 11, no. 1, Article ID e0146423, 2016.

[25] S. He, O. Wurtzel, K. Singh et al., "Validation of two ribosomal RNA removal methods for microbial metatranscriptomics," Nature Methods, vol. 7, no. 10, pp. 807-812, 2010.

[26] E. Kopylova, L. Noé, and H. Touzet, "SortMeRNA: Fast and accurate filtering of ribosomal RNAs in metatranscriptomic data," Bioinformatics, vol. 28, no. 24, pp. 3211-3217, 2012.

[27] C. de Weerth, S. Fuentes, P. Puylaert, and W. M. de vos, "Intestinal microbiota of infants with colic: development and specific signatures," Pediatrics, vol. 131, no. 2, pp. e550-e558, 2013.

[28] M. Kankainen, L. Paulin, S. Tynkkynen et al., "Comparative genomic analysis of Lactobacillus rhamnosus GG reveals pili containing a human-mucus binding protein," Proceedings of the National Academy of Sciences of the United States of America, vol. 106, no. 40, pp. 17193-17198, 2009.

[29] F. P. Douillard, A. Ribbera, R. Kant et al., "Comparative Genomic and Functional Analysis of 100 Lactobacillus rhamnosus Strains and Their Comparison with Strain GG," PLoS Genetics, vol. 9, no. 8, Article ID e1003683, 2013. 

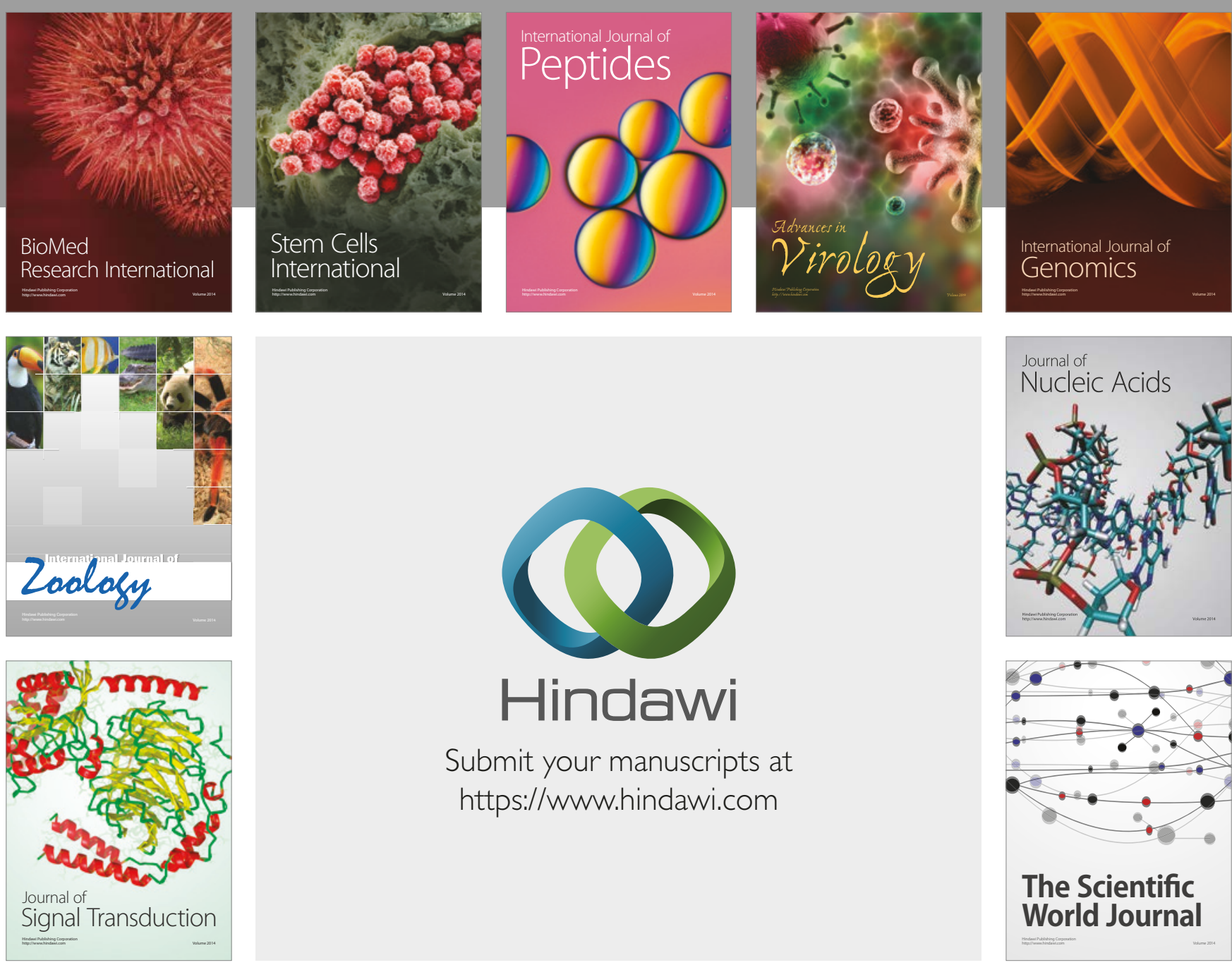

Submit your manuscripts at

https://www.hindawi.com
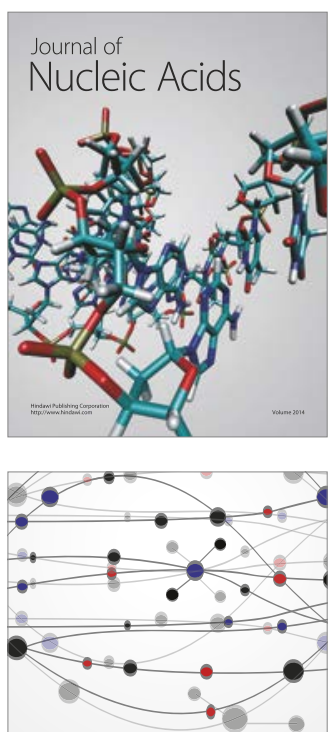

The Scientific World Journal

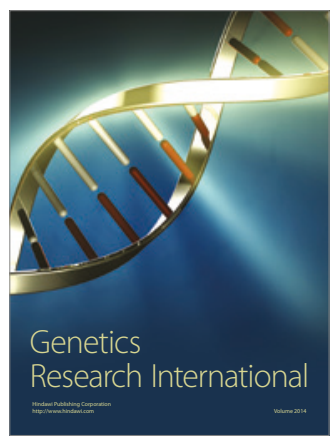

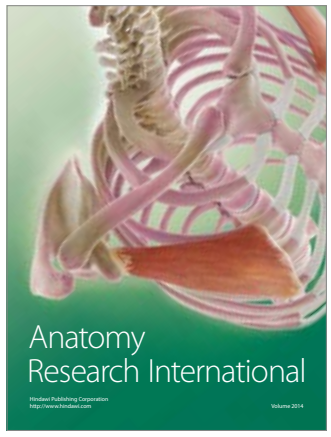

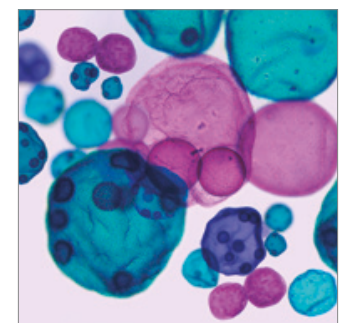

International Journal of Microbiology
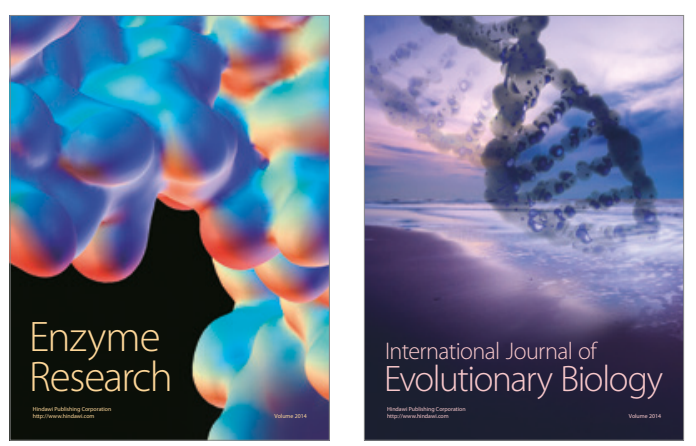
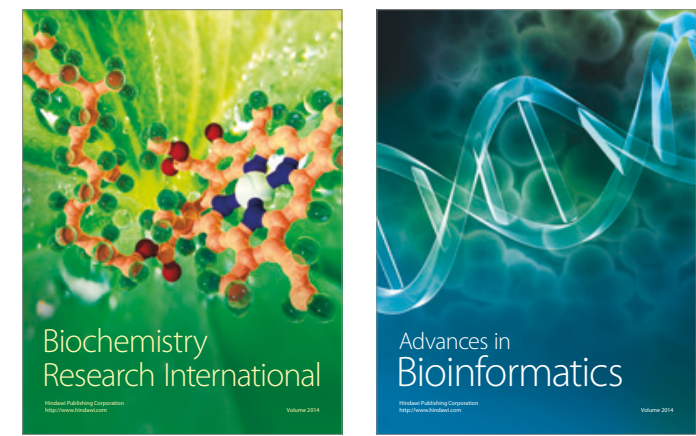

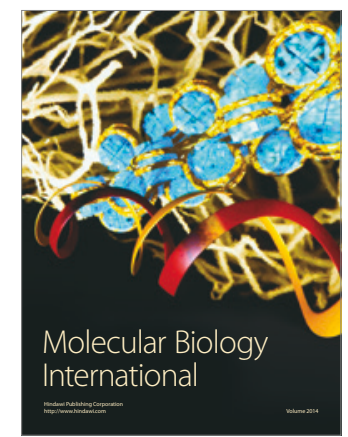

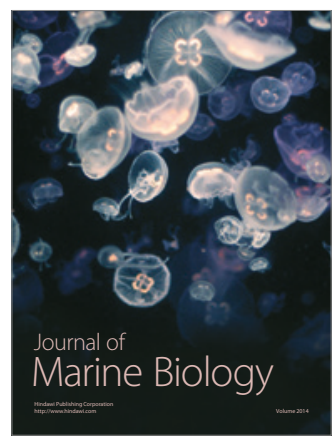

